\title{
Stability of multiple access communication networks
}

\author{
VINOD SHARMA \\ Department of Electrical Engineering, Indian Institute of Science, Bangalore \\ 560012 , India
}

\begin{abstract}
The problem of stability of different multiple access schemes in computer networks is discussed. The results for the main multiple access schemes - slotted ALOHA, controlled ALOHA, CSMA, CSMA/CD, polling systems and collision resolution algorithms are surveyed. The techniques used for the stability of these systems have been emphasized.
\end{abstract}

Keywords. Multiple access schemes; communication networks; channel access; stability.

\section{Introduction}

In data communications several resources usually share a common channel of communication. Traditionally, in telephone or radio networks time division multiple access (TDMA) or frequency division multiple access (FDMA) has been used to access the channel. These methods of channel access are efficient if the number of users is not very large and/or the communication traffic is not very bursty. But with the advent of large-scale use of computers it was felt that dedicating a time slot or a frequency band to each user is not very efficient because in such environments both these assumptions are generally violated. This could lead to unusually large delay in the communication and the channel may remain idle for a large fraction of time. This led researchers to look for new methods to share a channel.

The earliest new scheme for sharing channels was ALOHA. An improvement over it was slotted ALOHA. Since then a large number of techniques have been developed. Good reviews are available in Sachs (1988), Bertsekas \& Gallagher (1987). The purpose of this paper is to explain the main methods of multiple access that have been developed over the years and, more importantly, to present the most useful techniques that have been used to prove the stability of the channel under these methods.

We shall model the multiple access system as a stochastic system where the input traffic generated at the users for transmission is viewed as a stochastic process. In the literature the term stability has been used to imply different things. For us, the stability would usually mean the existence of stationary distributions of some system parameters of interest (e.g. queue lengths, delays) and the convergence to stationary distributions starting from any initial distribution. But we shall also briefly present other forms of stability like existence and convergence of moments of system parameters, structural stability, boundedness of sample paths of system parameters etc.

The paper is planned as follows. Section 2 describes the general multiple access 
system and the assumptions made. A unified stability analysis is given in $\S 3$ for the slotted ALOHA and TDMA systems. Section 4 discusses the controlled ALOHA system. First the instability of the infinite user model is proved and then various schemes to stabilize it have been mentioned. Again, in $\S 5$ for CSMA and CSMA/CD the instability of the infinite user model is shown and then some work concerning its stabilization has been described. Section 6 discusses the polling systems and $\S 7$ the collision resolution schemes. Section 8 briefly mentions some hybrid schemes which combine the random access and reservation schemes. Methods used to prove the stability of the various systems have been highlighted, the ergodicity of appropriate Markov chains being the predominant theme.

\section{Multiple access system}

There are $1<M \leqslant \infty$ users which share a common channel to communicate with each other. The messages are assumed packetized and usually having fixed packet lengths. The time axis will at times be slotted, i.e., it will be divided into slots of fixed size sufficient to send a packet if the packets are of the same lengths. In the slotted case the transmission of a packet can start only at the beginning of a slot. A user sends at most one packet at a time if it has something to transmit. The users will be assumed to have finite or infinite buffers to store the packets until successful transmission. When $M=\infty$, each user will be assumed to have a single buffer. If the transmission times of two or more packets overlap then the transmission is unsuccessful and the transmitters will send those packets again. On successful transmission the packets are removed from the buffers. It is assumed that the users can detect a collision or a successful transmission. The exact details of different multiple access methods will be presented at the appropriate places.

The stability of multiple access systems is generally studied either with $M<\infty$ and each user having an infinite buffer or with $M=\infty$. Both of these systems are idealization of the real systems where $M<\infty$ and the buffers at each user are finite. The relations between the stability for $M<\infty$ and $M=\infty$ assumptions have been studied by Paterakis et al (1987).

\section{Slotted ALOHA and TDMA}

In this section we present a technique by Loynes (1962) which has been used to demonstrate the stability of many systems. The advantage of this method is that it is quite intuitive and gives the existence of and convergence to stationary distributions under general stationary assumptions. We first explain the method in general and then apply it to slotted ALOHA and TDMA systems.

Let $\left\{x_{k}\right\}$ be a sequence of stationary random vectors with values in $R^{n}$ and let $\left\{z_{n}\right\}$ be a sequence with values in $R^{m}$ defined by (we call $\left\{x_{n}\right\}$ the input sequence and $\left\{z_{n}\right\}$ the state sequence)

$$
z_{n+1}=f\left(z_{n}, x_{n}\right)
$$

where $f$ is a measurable function. The following is Loyne's lemma. 
Lemma 1. If $f(z, x)$ is a nonnegative monotonically nondecreasing function in the first variable $z$ for each $x$ and is also continuous in $z$ then (1) has a stationary solution.

Proof. Let us denote by $z_{k}^{-n}$ the state value at time $k$ if the system started at time $-n$ with initial state 0 . Then it is easy to see that

$$
z_{k}^{-n-2} \geqslant z_{k}^{-n-1} \geqslant z_{k}^{-n} \quad \text { a.s. }
$$

Hence as $n \rightarrow \infty, z_{k}^{-n}$ converges almost surely (a.s.) to a limit $z_{k}$. It can easily be shown that the $\left\{z_{k}\right\}$ sequence so obtained is stationary and satisfies (1) a.s.

Remark 1. We can also show that if the input sequence $\left\{x_{m}\right\}$ is ergodic then so is the $\left\{z_{n}\right\}$ sequence obtained in the above lemma. Of course, it is possible that the limit sequence $z_{k}=\infty$ a.s. Therefore, to obtain an a.s. finite sequence we need to have extra conditions which we mention when we consider the particular systems.

Remark 2. It is easy to see that the distribution of $z_{k}^{-n}$ is the same as that of $z_{n+k}^{0}$. Therefore, since $z_{k}^{-n} \stackrel{W}{\rightarrow} z_{k}$ in the above lemma, we also have $z_{n+k}^{0} \stackrel{W}{\rightarrow} z_{k}$ as $n \rightarrow \infty$. Thus if the system starts empty at time 0 , then it converges in distribution to $z_{k}$. This can be shown for all finite dimensional distributions also.

Remark 3. The above lemma does not guarantee the uniqueness of the stationary distributions. But it can be shown that if more than one stationary distribution exists then the sequence obtained in lemma 1 is stochastically the smallest stationary distribution of (1).

Remark 4. The condition of monotonicity in the above lemma is quite strong and is not satisfied in many computer network systems.

Many practical systems can be modelled more appropriately to have periodic inputs, i.e., the finite dimensional distributions of $\left\{x_{n}, x_{n+1}, \ldots, x_{n+m}\right\}$ and $\left\{x_{n+d}, x_{n+1+d}, \ldots\right.$, $\left.x_{n+m+d}\right\}$ are the same for some integer $d \geqslant 1$ and for all $n$ and $m$. For this periodicity, we show now that the state equation $\left\{z_{n}\right\}$ in (1) has periodic stationary distributions with period $d$ under the assumptions of lemma 1.

Under the assumption of periodicity,

$$
z_{k}^{-n} \stackrel{\text { dist }}{=} z_{k+d}^{-n+d}
$$

where $X \stackrel{\text { dist }}{=} Y$ indicates that the random variables $X$ and $Y$ have the same distribution. Since $z_{k}^{-n} \stackrel{\underline{W}}{\rightarrow} z_{k}$ and $z_{k+d}^{-n+d} \stackrel{W}{\rightarrow} z_{k+d}, z_{k}$ and $z_{k+d}$ have the same distribution. The same can be said for all finite dimensional distributions. Hence, we can also show that if the system starts empty at time $n=0$, it converges to these periodic stationary distributions. Similar ideas have been expressed in Rolski (1981) and Bambos \& Walrand (1989) also.

Now we apply the above results on a multiple access system which has been analysed in Prathima (199'). This is a new scheme for multiple access that combines the advantages of slotted ALOHA and TDMA. The results for stationary and periodic stationary cases for the slotted ALOHA and TDMA will emerge as special cases.

We assume there are $1<M<\infty$ users each having infinite buffer to store the packets. The packets are of fixed size and the time axis is slotted. The $M$ users are 
divided into $L$ groups $G_{1}, G_{2}, \ldots, G_{L}$ (they don't have to be disjoint). Each slot is assigned to one of the groups in a periodic fashion. We take the period as $d$ slots. The question of how to form the groups, how large $d$ should be and how many slots should be assigned to a group in a period is an optimization problem which depends upon the traffic rates of different users. This problem is addressed in Prathima (1991) but presently we assume that the assignment has been made. In the slot assigned to a group, all the users which have packets to transmit at the beginning of that slot transmit a packet with a certain probability. If more than one packet gets sent then there is a collision and those packets will be tried again in the next assigned slot.

We use the following notation:

$k$ th slot $=$ time interval $(k, k+1)$

$x_{k}(i) \quad=$ number of new packets generated in the $(k-1)$ slot at the user $i$;

$t_{k}(i)=1$, if $i$ th user would transmit a packet in the $k$ th slot if it has, a packet at time $k$ $=0$, otherwise;

$z_{k}(i) \quad=$ queue length at the $i$ th user at time $k$ without including $x_{k}(i)$;

$1_{A} \quad=$ indicator function of set $A$;

$y_{k}(i) \quad=1$, if the $i$ th user has a successful transmission in the $k$ th slot $=0$, otherwise.

Then the system equation can be written as

$$
z_{k+1}=z_{k}+\left(x_{k}-y_{k}\right)
$$

where $z_{k}=\left(z_{k}(1), z_{k}(2), \ldots, z_{k}(M)\right)$ and similarly for $x_{k}$ and $y_{k}$. We can write this equation as

$$
z_{k+1}=f\left(z_{k}, x_{k}, t_{k}\right)
$$

The random variables $t_{k}$ take care of the assignment of the slots to different groups.

We can see that the function $f$ is monotonically nondecreasing in $z$ and is nonnegative. Thus, if $\left\{\left(x_{k}, t_{k}\right)\right\}$ is a periodic stationary process, then there exists a periodic stationary distribution of $\left\{z_{k}\right\}$ and starting at time $t=0$ with $z_{k}=0$, the finite dimensional distributions of $\left\{z_{k}\right\}$ converge to the periodic stationary distributions. For the finiteness of the stationary distributions (proper random variables) we need extra conditions on the rate of the input traffic. It can be shown, as in Sharma (1988), that if $E x_{k}<\infty, \Sigma_{k=1}^{d} E\left(x_{k}-y_{k}\right)<0$ and the input traffic $\left\{\left(x_{k}, t_{k}\right)\right\}$ is ergodic, the periodic stationary distributions of $\left\{z_{k}\right\}$ are proper. Also, as in Sharma (1988), it can be shown that if the system starts at $t=0$ with nonzero finite a.s. initial state then the random variables $\left\{z_{k}\right\}$ remain bounded a.s. Other remarks in Sharma (1988) remain relevant for this system as well.

It is easy to see that if $\left\{t_{k}\right\}$ is stationary then the above system becomes slotted ALOHA with periodic input, if $\left\{\left(x_{k}, t_{k}\right)\right\}$ is stationary then it is slotted ALOHA with stationary input, if $\sum_{i=1}^{M} t_{k}(i)=1$ for all $k$ and $t_{k}$ is deterministic then it is TDMA with periodic input.

Various other results have been obtained on the slotted ALOHA and TDMA systems. Slotted ALOHA with a finite number of users and infinite buffers has been studied by Saadawi \& Ephremides (1981) by forming two coupled Markov chains. Stability using Markov chains has also been shown by Tsybakov \& Mikhailov (1979). Both these papers assume the input traffic $\left\{x_{n}\right\}$ to be i.i.d. Within this framework most of the ergodicity and nonergodicity results have been summarized in Szpankowski (1986). 
For nonhomogeneous, nonperiodic input traffic, analysis has been provided in Sharma (1987). A comparative analysis of TDMA and FDMA is provided in Rubin (1979). The structural stability of slotted ALOHA has been studied in Nelson (1984) and Onozato $\&$ Noguchi (1985) via catastrophe theory. That the slotted ALOHA system with $M=\infty$ and unbuffered users is unstable for all input traffic rates has been proved by Rosenkrantz \& Towsley (1983) by martingale techniques. We present this result in detail in the next section.

\section{Controlled ALOHA}

In the last section we studied the slotted ALOHA system in which the retransmission probabilities remain constant. As mentioned there, it has been shown in Rosenkrantz \& Towsley (1983 and the earlier references therein) that for an infinite user model the slotted ALOHA system is unstable for any positive total input traffic, i.e., it has been shown that the total number of blocked users goes to infinity with probability 1 . This result, which was first noticed in the mid-seventies, has evoked a lot of research to control the retransmission probabilities of the users so that the system becomes stable for some nonzero input traffic (see Hajek 1985, Merakos \& Kazakos 1985, Bertsekas \& Gallagher 1987). The methods used for the study of such systems are quite different from those in the last section. In Merakos \& Kazakos (1985) and Hajek (1985) stochastic approximation methods have been used. Theory of Markov chains and large deviations has been used in Lim \& Meerkov (1987). Large deviation results have been used in Cottrell et al (1983) also. Martingale techniques have been used in Goodman et al (1988) to prove the stability of exponential backoff schemes. In $\S 4.1$ we show the above mentioned instability of slotted ALOHA for $M=\infty$. Section 4.2 will present a control scheme to stabilize the slotted ALOHA under these circumstances.

\subsection{Instability of infinite user slotted ALOHA}

We assume that the number of users $M=\infty$, each having a single buffer. Thus if a user has a packet stored then it cannot generate any more packets. Let $\left\{x_{k}\right\}$ be a sequence of i.i.d. random variables where $x_{k}$ is the number of new packets generated in the system in the $(k-1)$ th slot. Let $z_{k}$ represent the number of blocked users at time $k$, i.e., number of users which have packets that have been transmitted unsuccessfully earlier. A new packet generated in slot $k-1$ is transmitted in slot $k$ with probability 1 . A blocked user transmits its packet in the next slot with probability $p$. Then the $\left\{z_{k}\right\}$ sequence is a Markov chain with transition probabilities $P_{i j}$ given as below [in the following $P\left(x_{k}=i\right)=\lambda_{i}$ ],

$$
\begin{aligned}
P_{00} & =\lambda_{0}+\lambda_{1} ; \quad P_{01}=0 ; \quad P_{0 j}=\lambda_{j} ; \\
P_{i, i-1} & =\lambda_{0} i p(1-p)^{i-1}, \quad i \geqslant 1, \\
P_{i i} & =\lambda_{1}(1-p)^{i}+\lambda_{0}\left(1-i p(1-p)^{i-1}\right), \quad i \geqslant 1, \\
P_{i, i+1} & =\lambda_{1}\left(1-(1-p)^{i}\right), \quad i \geqslant 1, \\
P_{i j} & =\lambda_{j-1}, \quad j \geqslant i+2, \quad i \geqslant 1 .
\end{aligned}
$$

The following theorem has been used in Rosenkrantz \& Towsley (1983) to show the transience of Markov chain $\left\{z_{k}\right\}$. 
Theorem 1. If an irreducible, aperiodic Markov chain $\left\{z_{k}\right\}$ admits a nonconstant, nonnegative, bounded function $f$ on its state space $\{0,1,2, \ldots\}$ such that

$$
E\left[f\left(z_{k+1}\right) \mid z_{k}\right] \leqslant f\left(z_{k}\right),
$$

then the Markov chain is transient.

When $0<\lambda_{0}<1$ then $\left\{z_{k}\right\}$ is aperiodic. We further assume that $\lambda_{0}+\lambda_{1}<1$, otherwise the chain will be ergodic. Under these conditions a function $f$ has been constructed in Rosenkrantz \& Towsley (1983) which satisfies the conditions of theorem 1 and hence $\left\{z_{k}\right\}$ is transient.

\subsection{Controlled ALOHA}

We have seen in $\S 4.1$ that for the infinite user case the slotted ALOHA system is unstable if the retransmission probability $p$ is kept fixed. This happens because if at any time the number of blocked users increases, since $p$ is fixed, the probability that only one user transmits is very low. Therefore, as new packets are generated, the number of blocked packets keeps increasing with a high probability. Hence, attempts have been made to adaptively change $p$ as a function of the number of blocked users $z_{k}$ so that the probability of successful transmission remains high (Merakos \& Kazakos 1985; Hajek 1985; Lim \& Meerkov 1987; Tsitsklis 1987).

Now we present a scheme, given in Tsitsklis (1987) to stabilize the slotted ALOHA. As in $\$ 4.1, x_{k}$ is the number of new packets generated in slot $k-1$ and $z_{k}$ is the number of blocked users at time $k$ (beginning of slot $k$ ). Let $y_{k}$ be the number of attempted transmissions in slot $k$. At the beginning of each slot every station has an estimate $\hat{z}_{k}$ of the backlog, which it updates in each slot as below (the users get the feedback from the channel whether a slot was empty, there was successful transmission or a collision).

$$
\begin{array}{ll}
\hat{z}_{k+1}=\max \left\{1+\hat{z}_{k}-1+\hat{\lambda}\right\}, & \text { if } Y_{k}<2, \\
\hat{z}_{k+1}=\hat{z}_{k}+[1 /(e-2)]+\hat{\lambda}, & \text { if } Y_{k} \geqslant 2,
\end{array}
$$

where $\hat{\lambda}$ is the estimate of $\lambda$ at each user, $\lambda$ being the expected value of $x_{k}$. Actually, $\hat{\lambda}$ can be replaced by $e^{-1}$ and we can still obtain the stability and maximum throughput. The motivation for the updates of $z_{k}$ is provided in Bertsekas \& Gallagher (1987) and Rivest (1987).

We define $S_{k}=\left(z_{k}, \hat{z}_{k}\right)$. Then under the condition of $\left\{x_{k}\right\}$ being i.i.d., $S_{k}$ is a Markov chain. Now using the techniques of Hajek (1982) a Lyapunov function can be constructed to prove the following theorem.

Theorem 2. If $0<\hat{\lambda} \leqslant e^{-1}, 0<\lambda<e^{-1}$ and $\lambda \leqslant \hat{\lambda}$, then the Markov process $S_{k}$ defined above is geometrically ergodic (Tsitsklis 1987).

In the schemes mentioned in Merakos \& Kazakos (1985), Hajek (1985), Tsitsklis (1987) and Rivest (1987) each station has to continuously monitor the channel and update the backlog estimates etc, to follow the algorithm and their estimates should agree with each other for stability. To overcome this disadvantage, other algorithms have been proposed to control the slotted ALOHA (Aldous 1987; Hastad et al 1987; Goodman et al 1988). In Aldous (1987) it has been shown that for $M=\infty$ the Markov chain of backlogs $\left\{z_{n}\right\}$, when the retransmission probabilities $p$ of the users are 
exponentially decreased, is transient. In Goodman et al (1988), for number of users $M<\infty$, each with infinite buffer, under the exponential backoff, the Markov chain of queue lengths at each station has been shown to be positive recurrent if the traffic rate is below certain limits. These results have been generalized in Hastad et al (1987). Of course for finite user models the uncontrolled slotted ALOHA itself is stable. But the results of Hastad et al (1987) show that by controlling the retransmission probabilities we can increase the maximum stable throughput of the system.

The possibility of successful transmission of more than one packet in a slot has been considered in several papers (this is the "capture" effect). For such a slotted ALOHA network, control algorithms have been analysed in Ghez et al (1989).

The delay analysis of controlled ALOHA has been considered in Thomopoulos \& Sunder (1987) and Clare (1986).

\section{CSMA and CSMA/CD}

The multiple access scheme CSMA was first proposed and analyzed by Kleinrock \& Tobagi (1975). Its stability and control was discussed in Tobagi \& Kleinrock (1977). Since then a large number of papers have been devoted to this subject (Bertsekas \& Gallagher 1987). This scheme has proved very efficient when the propagation delays between different users are much less than the transmission times of the packets. An improvement over CSMA is CSMA/CD (Bertsekas \& Gallagher 1987; Tobagi \& Hunt 1987; Sachs 1988).

In CSMA, $M \leqslant \infty$ users share a common channel where each user can sense whether the channel is idle or busy. Whenever a user has a packet to send it first senses the channel for sometime (the time depends upon the propagation delays). If channel is free during this time then it sends its packet with probability $0 \leqslant p \leqslant 1$. If the channel is busy then it reschedules its sensing of the channel. When it next senses the channel depends upon the protocol assumed. Even after sensing that the channel is free, there is a possibility of a collision. In CSMA the user sends the complete packet even if there is a collision. But in CSMA/CD (CD stands for collision detection) after it senses a collision, the user aborts the transmission and hence frees the channel earlier than in CSMA.

\subsection{Instability of CSMA and CSMA/CD}

In this section we prove that the infinite user CSMA and CSMA/CD channels are unstable. The analysis follows Medith \& Lea (1983) where the Markov chain of blocked users is formed and is shown to be transient by using theorem 1 .

There are $M=\infty$ users each with a single buffer. The time axis is partitioned into minislots of lengths which equal the one-way propagation delay of the whole channel. The data packets are of fixed length and require a transmission time of $T>1$ minislots. In nonpersistent CSMA, if a user has a packet ready for transmission, it senses the channel. If the channel is sensed idle, it transmits the packet in the next slot. If it is found busy, the user schedules its transmission for some future minislot according to a probability distribution. In that slot if it again senses the channel busy it repeats the procedure. A blocked user senses the current slot with probability $0<f \leqslant 1$ and thereupon proceeds as above. The nonblocked users are assumed to generate new packets with Poisson distribution and mean rate $\lambda$ packets/minislot. 
The actual time taken in transmission for CSMA is $1+T$ minislots whether the transmission is a success or a failure (collision). Between two successive transmissions there is at least one idle slot. We consider the backlogged process at the instants of the end of the first idle minislot after a transmission and denote by $z_{i}$ the number of blocked users at these instants. This is a Markov chain. The transition probability of this Markov chain can be easily calculated under the above assumptions. Then, using theorem 1 , the following result can be obtained.

Theorem 3. The Markov chain $\left\{z_{n}\right\}$ of block users at the embedded points is transient (Medith \& Lee 1986).

A similar result for CSMA/CD has also been obtained in Medith \& Lea (1983). As for slotted ALOHA, this instability result has led researchers to obtain control schemes for CSMA and CSMA/CD also. We consider some of these results in the next section.

The delay analysis for CSMA and CSMA/CD has been studied in Beuerman \& Coyle (1988) and Tobagi (1982). Matrix geometric methods have been used in Beuerman \& Coyle (1988), while Tobagi (1982) uses Z-transform techniques.

\subsection{Control of CSMA and CSMA/CD}

To stabilise the CSMA and CSMA/CD adaptive algorithms changing the retransmission probabilities etc. have been proposed (Tobagi \& Kleinrock 1977; Medith \& Lea 1983; Rubin 1983; Coyle \& Liu 1985) just as for slotted ALOHA. Medith \& Lea (1983) consider only the slotted system, while Coyle \& Liu (1985) consider only the unslotted (continuous time) system and both cases are considered by Rubin (1983).

Medith \& Lea (1983) control CSMA and CSMA/CD by varying the retransmission probabilities $f$ of the blocked users as $f=\alpha / n$ where $n$ is the backlog. Of course $n$ will not be known to the users and hence will have to be estimated. The estimation schemes which have been used for controlling slotted ALOHA can be useful in this case also (see details later). Under the adaptive scheme of $f=\alpha / n$ Medith \& Lea (1983) have shown the ergodicity of the Markov chain $\left\{z_{n}\right\}$ formed in $\S 5.1$. For this they use the following Pake's lemma (Pake 1969).

Lemma 2. Let $\left\{x_{i}\right\}$ be an irreducible, aperiodic Markov chain whose state space is the set of nonnegative integers. Then, the following conditions are sufficient for the chain to be ergodic:

and

$$
\left|E\left[x_{i+1}-x_{i} \mid x_{i}=n\right]\right|<\infty,
$$

$$
\limsup _{n \rightarrow \infty} E\left[x_{i+1}-x_{i} \mid x_{i}=n\right]<0
$$

Using Lemma 2 it has been shown (Medith \& Lea 1983) that if

$$
\lambda T\left[1+(T+1)\left(1-e^{-(\alpha+\lambda)}\right)\right]<(\alpha+\lambda) T e^{-(\alpha+\lambda)},
$$

then Markov chain $\left\{z_{n}\right\}$ for CSMA is ergodic. A similar expression has been obtained for CSMA/CD also. From this condition, $\alpha$ can be appropriately chosen so as to obtain ergodicity for different arrival rates $\lambda$.

Coyle \& Liu (1985) have chosen the same adaptive scheme $f=\alpha / n$ and provide conditions for ergodicity, null-recurrence and transience of the Markov chain for the 
unslotted CSMA/CD. The system has been studied for exponential as well as phase type distributions and explicit closed form expressions obtained using the matrixgeometric techniques of Neuts (1981).

More recently, control schemes have been proposed which do not require the stations to know the total system backlog (Thomopoulos 1988; Cunningham \& Medith 1988). In (Thomopoulos 1988) minimum mean square error predictor has been used for the number of blocked users $z_{n}$ as below (the updating is done at the end of the idle period, the successful transmission period and the collision period).

$$
\begin{array}{rlrl}
\hat{Z}_{n+1} & =\hat{Z}_{n}-\alpha /\left(\alpha+\lambda_{\text {eff }}\right), & & \text { if successful transmission, } \\
& =0 \quad, \text { if idle period, } \\
& =\lambda_{\text {eff }} /\left[1-\left(\alpha+\lambda_{\text {eff }}\right) /\left(\exp \left(\alpha+\lambda_{\text {eff }}\right)-1\right)\right], \quad \text { if collision, }
\end{array}
$$

where $\lambda_{\text {eff }}=\lambda$ (length of the period).

Then the retransmission probability $f$ is updated as

$$
f_{n+1}=\min \left(\beta, \alpha / \hat{Z}_{n+1}\right) \text {. }
$$

If the traffic intensity $\lambda$ is not known then an estimate of $\lambda$ is also provided in Thomopoulos (1988). Under these estimates the following result for stability has been obtained. The packet length is taken as 1 slot.

\section{COROLLARY.}

The maximum stable throughput obtained by 1-persistent CSMA, under the above adaptive scheme, is the solution of

$$
(1-x) e^{-x}=1 /(1+\tau)
$$

where $\tau$ is the time required by the stations to sense the channel being idle. For $\tau \ll 1$, $\lambda_{\max } \approx 1-\sqrt{2} \tau$. For CSMA/CD the maximum stable throughput is obtained by solving the equation

$$
(1-x) e^{-x}=1 /\left(1+\tau / \tau_{c}\right),
$$

where $\tau_{c}$ is the fraction of a slot used in collision detection.

Thomopoulos (1988) defines the stability in the following way: the average rate of successful transmission should be maintained and the expected delay should remain bounded as the number of users sharing the channel approaches infinity.

The control scheme used in Cunningham \& Medith (1988) applies stochastic approximation techniques, following Merakos \& Kazakos (1985) and Hajek (1982, 1985).

\section{Polling schemes}

In this scheme, the different users sharing the channel are polled in a certain order for using the channel. The polling in a physical system can be done in various ways. One common method is by sending a token to different stations whenever the channel is free. When a station/user is polled, it may or may not be allowed to send more than one packet/message at a time. Even TDMA can be considered a polling system. 
But in general, in a polling system the disadvantage of TDMA, in having the channel idle for a long time when there are a large number of users, is not there. Therefore, these schemes can be very efficient in local area networks (Sachs 1988; Bertsekas \& Gallagher 1987). Henee, polling systems have been extensively studied. Since rather exhaustive surveys are available for these systems (see Takagi 1986, 1990) we shall limit ourselves to only the references which are directly relevant for the stability of the polling systems.

The polling systems have been studied under various assumptions. The number of stations $M<\infty$. The stations can have finite or infinite buffers. The time axis can be slotted or unslotted. The number of messages allowed for transmission at a time from a user can be one, $k \geqslant 1$, exhaustive (i.e. all packets queued at the station) or gated (with various restrictions). The walk time, i.e., the time to poll from one user to another or the time a token takes to reach from one user to another can be zero, deterrministic or random. If the walk time is zero then the stability of polling systems can be studied in the generality of $\S 3$. If walk time is nonzero then the stationary distributions in such generality may not exist. Therefore, in this section we consider the stability of polling systems where each user has an infinite buffer (stability problem does not arise for the finite buffer case), the walk times are nonzero and the message generation sequences are Poisson processes (for the unslotted case) or i.i.d. sequences (for the slotted case).

The main references for stability of the polling systems are Eisenberg (1972), Hashida (1972), Konheim \& Meister (1974), Zhdanov \& Saksonov (1979) and Swartz (1980). A nice survey emphasising the performance and technological aspects of token rings is given is Bux (1989). In Konheim \& Meister (1974) and Swartz (1980) the time axis is considered slotted with i.i.d. input sequences while in Eisenberg (1972), Hashida (1972) and Zhdanov \& Saksonov (1979) the system is unslotted. The arrival processes at each user are Poisson in Hashida (1972) and Eisenberg (1972), while more general renewal arrival process at each user is considered in Zhdanov \& Saksonov (1979). But the criterion of stability, which is ergodicity of appropriate Markov chains in Hashida (1972) and Eisenberg (1972), is the existence of finite limit of expected values of certain system parameters (for Poisson input the results in Zhdanov \& Saksonov (1979) reduce to that of others).

We present, in the following, the results of Zhdanov \& Saksonov (1979). Eisenberg (1972), Hashida (1972), Konheim \& Meister (1974) and Swartz (1980) have used Laplace-Stieltjes transform techniques, which involve many tedious manipulations. Also, as mentioned above, Zhdanov \& Saksonov (1979) do convey something in the case of general renewal input process also.

There are $1<M<\infty$ users each with an infinite buffer. The input messages generated at the users are independent renewal processes. The interarrival time at user $i$ has mean $0<\alpha_{i}<\infty$ and the service time has general distribution with mean $0<\beta_{i}$. Walk time from user $i$ to user $i+1$ is random having a general distribution with finite mean $0<\gamma_{i}<\infty$. All random variables are independent of each other. We consider in the following only the gated system although the exhaustive service system has also been treated by Zhdanov \& Saksonov (1979).

For the system described above, it is easy to show that

$$
t_{i k}=\left[\sum_{j=1}^{M} \gamma_{j}\right] / \alpha_{i}+\left[\sum_{j=1}^{i-1} t_{j k} \beta_{j}+\sum_{j=i+1}^{M} t_{j k-1} \beta_{j}\right] / \alpha_{i},
$$


where $t_{i k}$ is the expected value of the total time the server spends at user $i$ in the $k$ th cycle (i.e., the $k$ th time it comes to user $i$ ). This can be rewritten as

where,

$$
t_{k}=(I-A)^{-1} B t_{k-1}+(I-A)^{-1} \gamma,
$$

$$
\begin{aligned}
t_{k} & =\left(t_{1 k}, t_{2 k}, \ldots, t_{M k}\right)^{T}, \\
\gamma & =\left(\sum_{i=1}^{M} \gamma_{i}\right)^{-1}\left(\beta_{1} / \alpha_{1}, \beta_{2} / \alpha_{2}, \ldots, \beta_{M} / \alpha_{M}\right)^{T}, \\
A & =\left[\begin{array}{cccc}
0 & 0 & \cdots & 0 \\
\rho_{2} & 0 & \cdots & 0 \\
\rho_{3} & \rho_{3} & 0 & 0 \\
\cdot & & & \\
\cdot & & & \\
\rho_{M} & \rho_{M} & \cdots & 0
\end{array}\right]
\end{aligned}
$$$$
B=\left[\begin{array}{ccccc}
\rho_{1} & \rho_{1} & \rho_{1} & \cdots & \rho_{1} \\
0 & \rho_{2} & \rho_{2} & \cdots & \rho_{2} \\
\cdot & & & & \\
\cdot & & & & \\
\cdot & & & & \\
0 & 0 & 0 & \cdots & \rho_{N}
\end{array}\right] \text {, }
$$

and

$$
\rho_{i}=\beta_{i} / \alpha_{i}
$$

Now, it is well-known that this recursive equation has a finite limiting value if and only if $(I-A)^{-1} B$ has all its eigenvalues in a unit circle in the complex plane. It has been shown in Zhdanov \& Saksonov (1979) that a sufficient condition for this is

$$
\sum_{i=1}^{M}\left(\beta_{i} / \alpha_{i}\right)<1
$$

The finiteness of the limiting value of $t_{i k}$ implies the finiteness of the limiting value of expected valued $\eta_{i k}$ (as $k \rightarrow \infty$ ) of the number of customers served in $k$ th cycle at user $i$.

If the arrival processes at the users are independent Poisson processes then the above result gives the ergodicity of the Markov chain of queue length processes formed at the instants of arrival of the server at ith user. Analysis in the following differs somewhat from that in Zhdanov \& Saksonov (1979) and provides a little stronger result which is equivalent to that obtained in Hashida (1972) and Eisenberg (1972). We denote $\left(z_{n}(1), z_{n}(2), \ldots, z_{n}(M)\right)$ by $z_{n}$, where $z_{n}(j)$ is the queue length at the $j$ th user when the server arrives at the ith queue (fixed in the following) in the $n$th cycle. Then $\left\{z_{n}\right\}$ is an aperiodic, irreducible Markov chain. We show in the following that the above result on finiteness of the limit of the expected values implies that $\left\{z_{n}\right\}$ is ergodic.

It can be easily seen that $z_{n}(j), j \neq i$ is a.s. dominated by the queue length at user $j$ when the server visits $j$ either in $n$th cycle or in $(n+1)$ th cycle. Thus $\lim _{n \rightarrow \infty} \sup E\left[z_{n}(j)\right]<\infty$. Now we define a random sequence $\left\{s_{n}\right\}$ by

$$
S_{n}=\sum_{j=1}^{M} z_{n}(j)
$$


Then $E s_{n}=\sum_{j=1}^{M} E\left[z_{n}(j)\right]$ and $\lim _{n \rightarrow \infty} \sup E\left(s_{n}\right)<\infty$. Since $\left\{z_{n}\right\}$ is irreducible, if $\left\{z_{n}\right\}$ is not an ergodic sequence then $P\left\{z_{n}=a\right\} \rightarrow 0$ for any vector $a=\left(a_{1}, \ldots, a_{M}\right)$. This implies that $P\left\{S_{n}=k\right\} \rightarrow 0$ for all $k \geqslant 0$. Therefore,

$$
\liminf _{n \rightarrow \infty} E s_{n}=\liminf _{n \rightarrow \infty} \sum_{k=0}^{\infty} P\left\{S_{n} \geqslant k\right\} \geqslant \sum_{k=0}^{\infty} \lim _{n \rightarrow \infty} P\left\{S_{n} \geqslant k\right\}=\infty
$$

Hence $\left\{z_{n}\right\}$ is an ergodic Markov chain.

We now mention a few more papers on the stability of the polling systems. The stability in these papers may not imply the ergodicity of any underlying Markov chain. An influential paper is by Kuehn (1979). Analysis of this kind has been followed up in Servi (1985) and lbe \& Chang (1989). See Takagi (1990) for many more references.

\section{Collision resolution algorithms}

We have seen in the earlier sections that for infinite user models, slotted ALOHA, CSMA and CSMA/CD are all unstable for any nonzero input traffic. Collision resolution algorithms (CRA) introduced in Capetanakis (1979) and Tsybakov \& Mikhailov (1978) were the first algorithms among the random multiple access methods which provide nonzero stable throughput. The currently available CRA have throughputs upto 0.487 which exceed the throughput of $1 / e$ available from controlled ALOHA. The IEEE special issue (IEEE Trans. Inf. Theory 1985) contains the history, most of the references to earlier literature and the state of the art till 1984 on these algorithms. We shall mention some more recent work at the end of this section.

The number of users $M=\infty$ and each user has a single buffer. The system is slotted and the packet lengths are fixed. Total system packet generation process is Poisson. A nonblocked binary stack algorithm is described as follows (Tsybakov 1985).

(i) If a station has a packet to transmit, then in the time interval from the packet's generation to its successful transmission, it assigns to this packet an integer $L_{n}$ in slot $n . L_{n}$ is called its packet level at time $n$. The packet is transmitted over the channel in slot $n$ if and only if $L_{n}=0$.

(ii) Every new packet is assigned level 0 .

(iii) If $L_{n}=0$ for a packet and there is collision in slot $n$ then $L_{n+1}=1$ with probability $1 / 2$, and $L_{n+1}=0$ otherwise.

(iv) If $L_{n} \geqslant 1$ for a packet and there is a collision in slot $n$ then $L_{n+1}=L_{n}+1$.

(v) If $L_{n} \geqslant 1$ and there is no collision in slot $n$ then $L_{n+1}=L_{n}-1$.

A generalization of the above algorithm is nonblocked $J$-ary stack algorithm in which the steps (iii) and (iv) in the above algorithm are changed to:

(iii)' If $L_{n}=0$ for a packet and there is a collision in slot $n$ then $L_{n+1}$ takes value $j$ with probability $P_{j}$, where $j \in\{0,1, \ldots, J-1\}$.

(iv) If $L_{n} \geqslant 1$ and there is a collision in slot $n$ then

$$
L_{n+1}=L_{n}+J-1 \text {. }
$$

The above algorithm has been modified to eliminate certain predictable collisions. The details are in Massey (1981, pp. 73-137). In another algorithm called blocked stack algorithm (Tsybakov \& Mikhailov 1978), the newly generated packets are not 
allowed to transmit immediately. If there has been a collision in the recent past, all the packets involved in that collision are first allowed to transmit. Only after their successful transmission, packets generated during that time transmit and then if there is a collision, these packets get into what is called a collision resolution period. The disadvantage of the blocked algorithm as compared to the nonblocked one, explained above, is that in the blocked case all the stations have to keep monitoring the channel throughout whether they have a packet to transmit or not. A further variation along the theme of collision resolution is the part and try algorithm (Tsybakov \& Mihailov 1980).

In the following we provide the stability results for the blocked stack algorithm. The analysis for the nonblocked is along the same lines. Assume that $x_{k}$ is the number of new packets generated in the system in the $k$ th slot. The sequence $\left\{x_{k}\right\}$ is assumed i.i.d. Also, let

$$
P\left\{x_{k}=m\right\}=\lambda^{m} e^{-\lambda} / m !, \quad m=0,1, \ldots
$$

The analysis can easily be extended to the general distributions. Let us denote by $\tau_{k}$ the length of a collision resolution period given that the collision multiplicity (number of packets involved in the initial collision) is $k$. Then, the following recursive equation holds.

$$
E\left[\tau_{k}\right]=P_{0}\left(1+E\left(\tau_{k}\right)\right)+\sum_{n=1}^{k} P_{n}\left(2+E\left(\tau_{k-n}\right)+E\left(\tau_{n}\right)\right)
$$

where $P_{l}=2^{-k}\left(\begin{array}{l}k \\ l\end{array}\right)$ and the initial conditions are $E\left[\tau_{0}\right]=E\left[\tau_{1}\right]=0$. This provides $E\left[\tau_{2}\right]=3 \cdot 5, E\left[\tau_{3}\right]=6$ etc. Also, it can be shown (Tsybakov \& Mikhailov 1978) that

$$
[(8 / 3)-(1 / 168)] k-2 \leqslant E\left[\tau_{k}\right] \leqslant(8 / 3) k-2 .
$$

We denote by $\xi_{n}$ the multiplicity of the $n$th conflict. Under the assumption of $\left\{x_{n}\right\}$ being i.i.d., it can be shown that $\left\{\xi_{n}\right\}$, and $\left\{\tau_{n}\right\}$ are homogeneous Markov chains. Using the results on the ergodicity of Markov chains similar to theorem 1 and lemma 2 , the following theorem can be obtained.

Theorem 4. Under the above assumptions the following hold (Tsybakov \& Mikhailov 1978)

(i) If $\lambda<\liminf _{k \rightarrow \infty}\left(k / E \tau_{k}\right)$ then $\left\{\xi_{n}\right\}$ is an ergodic Markov chain.

(ii) If $\lambda \leqslant \lim \inf _{k \rightarrow \infty} k / E \tau_{k}$ then $\left\{\xi_{n}\right\}$ is a recurrent Markov chain.

(iii) If $\lambda>\lim \sup _{k \rightarrow \infty} k / E \tau_{k}$ then $\left\{\xi_{n}\right\}$ is a transient Markov chain.

From the estimates given in (3), theorem 4 states that if $\lambda<3 / 8$ then $\left\{\xi_{n}\right\}$ is ergodic and if $\lambda \geqslant 3 / 8$ then it is recurrent. It has been shown (Tsybakov \& Mikhailov 1978) that if $\lambda<3 / 8$ then $\left\{\tau_{n}\right\}$ is also an ergodic Markov chain, that $\lim E \tau_{n}<\infty$ and that $\lim E \xi_{n}<\infty$.

Recently sharper asymptotic estimates have been obtained for the $E\left[\tau_{k}^{m}\right]$, for $m \geqslant 1$ (Jacquet \& Szpankowski 1989). The performance of the collision resolution algorithms can be improved somewhat if the channel feedback is used to estimate the collision multiplicity also (Cidon \& Sidi 1986). This estimate is then used to choose dynamically the $J$-parameter in a $J$-ary stack algorithm.

In a part and try algorithm (Tsybakov \& Mihailov 1980; Gallagher 1978) the 
collision resolution is done by allowing the colliding packets to transmit in order of their time of generation. This is done by splitting the time axis and allowing packets to be transmitted if they arrived in a certain subset of the time axis. In Mosely \& Humblet (1985) and Khasminskii (1989) the problem of splitting the time axis optimally has been addressed by forming a Markov decision problem.

The collision resolution algorithms discussed above have been in the framework when the channel propagation time is large. In local area networks (LAN), collisions and carriers can be sensed in a short time and, exploiting this feature, collision resolution algorithms for LAN have been studied in Georgiopoulos et al. (1986, pp. 185-214).

\section{Hybrid techniques}

As described in $\S 3$, random access schemes perform well when there are a large number of bursty users with light traffic and reservation based schemes like TDMA provide good performance when the number of users is small and traffic intensity is high. This has motivated many researchers to develop hybrid schemes combining random access and reservation techniques. Our scheme in $\S 3$ is motivated by the same idea. Several references to the hybrid schemes are provided in Sachs (1988). Also, several hybrid schemes are mentioned in Mehravari (1984) and it has been shown that under heavy load the TDMA is better than any random access scheme. Multiple access schemes with priority have been reviewed in de Moraes (1990).

An error in the first draft of this paper was pointed out by the reviewer.

\section{References}

Abramson N 1970 The ALOHA system - Another alternative for computer communications. In Proc. Fall Joint Computer Conf. (Montvale, NJ: AFIPS Press) vol. 37, pp. 281-285

Aldous D J 1987 Ultimate instability of exponential back-off protocol for acknowledgement based transmission control of random access communication channels. IEEE Trans. Inf. Theory IT-33: 219-223

Bambos N, Walrand J 1989 On queues with periodic inputs. J. Appl. Probab. 26: 381-389

Bertsekas D, Gallagher R 1987 Data networks (Englewood Cliffs, NJ: Prentice-Hall)

Beuerman S L, Coyle E J 1988 The delay characteristics of CSMA/CD networks. IEEE Trans. Commun. 36: $553-563$

Bux W 1989 Token ring local area networks and their performance. Proc. IEEE 77: 238-256

Capetanakis J I 1979 Tree algorithm for packet broadcast channels. IEEE Trans. Inf. Theory IT-25: 505-5i5

Cidon I, Sidi M 1986 Conflict multiplicity estimation and batch resolution algorithms. INFOCOM'86: (New York: IEEE Press) pp. 197-203

Clare L P 1986 Delay analysis of stable slotted ALOHA systems. INFOCOM'86 (New York: IEEE Press) pp. $10-19$

Cottrell M, Claude Fort J, Malgouyres G 1983 Large deviations and rare events in the study of stochastic algorithms. IEEE Trans. Autom. Control AC-28: 907-920

Coyle E J, Liu B 1985 A matrix representation of CSMA/CD networks. IEEE Trans. Commun. COM-33: 53-64

Cunningham G A, Medith J S 1988 Distributed retransmission controls for slotted nonpersistent and virtual time CSMA. IEEE Trans. Commun. 36: 685-691 
de Moraes L F M 1990 Priority scheduling in multiple access communication. In stochastic analysis of computer \& communication systems (ed.) H Takagi (Amsterdam: Elsevier Science Publishers)

Eisenberg M 1972 Queues with periodic service and changeover time. Oper. Res. 20: 440-451

Gallagher R G 1978 Conflict resolution in random access broadcast networks. In Proc. AFOSR Workshop on Commun. Theory Appl. pp. 74-76

Georgiopoulos M, Merakos L, Papatani-Kazakos P 1986 High performance asynchronous limited sensing algorithms for CSMA and CSMA/CD channels. In Local area and multiple access networks (ed.) R L Pickholtz (Rockville, MD: Computer Science Press) pp. 185-214

Ghez S, Verdu S, Schwartz S C 1989 Optimal decentralized control in the random access multipacket channel IEEE Trans. Autom. Control 34: 1153-1163

Goodman J, Greenberg A G, Madras N, March P 1988 Stability of binary exponential backoff. $J$. Assoc. Comput. Mach. 35: 579-602

Hajek B 1982 Hitting time and occupation time bounds implied by drift analysis with applications. $A d v$. Appl. Probab. 14: 502-525

Hajek B 1985 Stochastic approximation methods for decentralized control of multiaccess communications. IEEE Trans. Inf. Theory IT-31: 176-184

Hashida O 1972 Analysis of multiqueue. Rev. Elec. Commun. Lab. 20: 189-199

Hastad J, Leighton T, Rogoff B 1987 Analysis of backoff protocols for multiple access channels. Proc. 19th Annu. ACM Symp. on Theory of Comput. pp. 241-243

Ibe O C, Chang X 1989 Stability conditions for multiqueue systems with cyclic service. IEEE Trans. Autom. Control 33: 102-103

IEEE Trans. Inf. Theory 1985 vol. IT-31, Special issue on Random-Access Communications

Jacquet P, Szpankowski W 1989 Ultimate characteristics of interval searching algorithms for distributed systems. In Performance of distributed and parallel systems (eds) T Hasegawa, H Takagi, Y Takahashi (Amsterdam: Elsevier Science Publishers)

Khasminskii R Z 1989 Some optimal algorithms of random multiple access. Theory Probab. Appl. 34: 454-463

Kleinrock L, Tobagi F A 1975 Packet switching in radio channels: part I - Carrier sense multiple access models and their throughput - delay characteristics. IEEE Trans. Commun. Com-23: 1400-1416

Konheim A G, Meister B 1974 Waiting lines and times in a system with polling. J. Assoc. Comput. Mach. 21: $470-490$

Kuehn P J 1979 Multiqueue systems with nonexhaustic cyclic service. Bell Syst. Tech. J. 58: 671-698

Lim J T, Meerkov S M 1987 Theory of Markovian access to collision channels. IEEE Trans. Commun. COM-35: $1278-1288$

Loynes R 1962 The stability of a queue with non-independent interarrival and service times. Proc. Cambridge Philos. Soc. 58: 497-520

Massey J L 1981 Collision resolution algorithms and random access communications. In Multiuser communications systems (ed.) G Longo (CISM courses and lectures No. 265) (New York: Springer)

Medith J S, Lea C-T A 1983 Stability and optimization of the CSMA and CSMA/CD channels. IEEE Trans. Commun. COM-31: 763-774

Mehravari N 1984 TDMA in a random access environment, an overview. IEEE Commun. Mag. 22(11): 54-59

Merakos L, Kazakos D 1985 On the retransmission control policies in multiple access communication networks. IEEE Trans. Autom. Control. AC-30: 109-117

Mosely J, Humblet P A 1985 A class of efficient contention resolution algorithms for multiple access. IEEE Trans. Commun. COM-33: 145-151

Nelson R 1984 The stochastic cusp, swallowtail and hyperbolic umbilic catastrophes as manifest in a simple communications model. Performance'84 (Amsterdam: Elsevier Science Publishers)

Neuts M F 1981 Matrix geometric solutions in stochastic models (Baltimore: Johns Hopkins Univ. Press)

Onozato Y, Noguchi S 1985 On the thrashing cusp in slotted ALOHA systems. IEEE Trans. Commun. COM-33: 1171-1182

Pakes A G 1969 Some conditions for ergodicity and recurrence of Markov chains. Oper. Res. 117: 1059-1061

Paterakis M, Georgiadis L, Papatani-Kazakos P 1987 On the relation between the finite and the infinite population models for a class of RAA's. IEEE Trans. Commun. COM-35: 1239-1240

Prathima B V 1991 Stability and performance analysis of same multiple access and mobile cellular communication networks, MS thesis, Department of Electrical Engineering, Indian Institute of Science, Bangalore

Rivest R L 1987 Network protocol by Bayesian broadcast. IEEE Trans. Inf. Theory IT-33: 323-328

Rolski T 1981 Stationary random processes associated with point processes (New York: Springer-Verlag) 
Rosenkrantz W A, Towsley D 1983 On the instability of the slotted ALOHA multiaccess algorithm. IEEE Trans. Autom. Control AC-28: 994-996

Rubin I 1979 Message delay in FDMA and TDMA communication channels. IEEE Trans. Commun. COM-27: 516-536

Rubin I 1983 Synchronous and channel sense asynchronous dynamic group-random-access scheme for multiple access communications. IEEE Trans. Commun. COM-31: 1063-1076

Saadawi T N, Ephremides A 1981 Analysis, stability and optimization of slotted ALOHA with a finite number of buffered users. IEEE Trans. Autom. Control. 26: 680-689

Sachs S R 1988 Alternative local area network access protocols. IEEE Commun. Mag. 26(3): 25-44

Servi L D 1985 Capacity estimation of cyclic queues. IEEE Trans. Commun. COM-33: 279-282

Sharma V 1987 Stability analysis of slotted ALOHA with time varying input, NASA Tech. Report

Sharma V 1988 Stability and continuity for slotted ALOHA with stationary non-independent input traffic. J. Appl. Probab. 25: 797-807

Swartz G B 1980 Polling in a loop system. J. Assoc. Comput. Mach. 27: 42-59

Szpankowski W 1986 Stability, bounds and approximation in asymmetric buffered ALOHA-type systems. Proc. 25 th Conf. Decision and Control (New York: IEEE Press) pp. 1722-1727

Takagi H 1986 Analysis of polling systems (Cambridge, MA: MIT Press)

Takagi H 1990 Queueing analysis of polling models: An update. In Stochastic analysis of computer and communication systems (ed.) H Takagi (Amsterdam: Elsevier Science Publishers)

Thomopoulos S C A 1988 A simple and versatile decentralized control for slotted ALOHA, reservation ALOHA and local area networks. IEEE Trans. Commun. 36: 662-674

Thomopoulos S C A, Sunder R 1987 Delay analysis of the controlled ALOHA: exact analysis and bounds. IEEE INFOCOM'87 (New York: IEEE Press) pp. 26-33

Tobagi F A 1982 Distributions of packet delay and interdeparture time in slotted ALOHA and carrier sense multiple access. J. Assoc. Comput. Mach. 29: 907-922

Tobagi F A, Hunt V B 1987 Performance analysis of carrier sense multiple access with collision detection. In Advances in local area networks (ed.) Karl Kummerle, J O Limb, F A Tobagi (New York: IEEE Press)

Tobagi F, Kleinrock L 1977 Packet switching in radio networks, Part IV. Stability considerations and dynamic control in carrier sense multiple access. IEEE Trans. Commun. COM-25: 1103-1120

Tsitsklis J N 1987 Analysis of a multiaccess control scheme. IEEE Trans. Autom. Control AC-32: 1017-1029

Tsybakov B S 1985 Survey of USSR contributions to random multiple access communications. IEEE Trans. Inf. Theory IT-31: 143-165

Tsybakov B S, Mikhailov V A 1978 Free synchronous access of packets to a broadcast channel with feedback. Probl. Peredachi Inf. 14(4): 32-59

Tsybakov B S, Mikhailov V A 1979 Ergodicity of ALOHA system. Probl. Peredachi Inf. 15: 75-87

Tsybakov B S, Mihailov V A 1980 Random multiple access of packets: part and try algorithm. Probl. Peredachi Inf. 16: 65-79

Zhdanov V S, Saksonov E A 1979 Conditions of existence of steady state modes in cyclic queueing systems. Avtom. Telemekh. (Engl. Transl.) 40: 176-184 